\title{
ONLINE SELECTION OF ALTERNATING SUBSEQUENCES FROM A RANDOM SAMPLE
}

\author{
ALESSANDRO ARLOTTO, ${ }^{*}$ University of Pennsylvania \\ ROBERT W. CHEN, ${ }^{* *}$ University of Miami \\ LAWRENCE A. SHEPP ${ }^{* * *}$ AND \\ J. MICHAEL STEELE, ${ }^{* * * *}$ University of Pennsylvania
}

\begin{abstract}
We consider sequential selection of an alternating subsequence from a sequence of independent, identically distributed, continuous random variables, and we determine the exact asymptotic behavior of an optimal sequentially selected subsequence. Moreover, we find (in a sense we make precise) that a person who is constrained to make sequential selections does only about 12 percent worse than a person who can make selections with full knowledge of the random sequence.
\end{abstract}

Keywords: Bellman equation; online selection; sequential selection; prophet inequality; alternating subsequence

2010 Mathematics Subject Classification: Primary 60C05; 90 C40

Secondary 90C27; 90C39

\section{Introduction}

Given a finite (or infinite) sequence $\boldsymbol{x}=\left\{x_{1}, x_{2}, \ldots, x_{n}, \ldots\right\}$ of real numbers, we say that a subsequence $x_{i_{1}}, x_{i_{2}}, \ldots, x_{i_{k}}, \ldots$ with $1 \leq i_{1}<i_{2}<\cdots<i_{k}<\cdots$ is alternating if we have $x_{i_{1}}<x_{i_{2}}>x_{i_{3}}<x_{i_{4}}>\cdots$. When $\boldsymbol{x}$ is an element of the set of permutations $s_{n}$ of the integers $\{1,2, \ldots, n\}$, the study of the set of alternating permutations goes back to Euler (cf. Stanley (2010)).

Here we are mainly concerned with the length $a(\boldsymbol{x})$ of the longest alternating subsequence of $\boldsymbol{x}$. This function has recently been studied in Widom (2006), Pemantle (cf. Stanley (2007, p. 568)), and Stanley (2008). In particular, they considered the situation in which $\boldsymbol{x}$ is chosen at random from $\ell_{n}$. By exploiting explicit formulae for generating functions and delicate applications of the saddle point method, they were able to obtain exact formulae for the first two moments and to prove a central limit theorem. Specifically, if $\boldsymbol{x}$ is chosen according to the uniform distribution on the set of permutations $\wp_{n}$ and if $A_{n}:=a(\boldsymbol{x})$ denotes the length of the longest alternating subsequence of $\boldsymbol{x}$, then, for $n \geq 4$, we have

$$
\mathrm{E}\left[A_{n}\right]=\frac{2 n}{3}+\frac{1}{6} \quad \text { and } \quad \operatorname{var}\left[A_{n}\right]=\frac{8 n}{45}-\frac{13}{180} .
$$

Received 10 May 2011; revision received 1 July 2011.

* Postal address: Wharton School, Department of Operations and Information Management, Huntsman Hall 527.2, University of Pennsylvania, Philadelphia, PA 19104, USA. Email address: alear@wharton.upenn.edu

** Postal address: Department of Mathematics, University of Miami, Coral Gables, FL 33124, USA.

Email address: chen@math.miami.edu

*** Postal address: Wharton School, Department of Statistics, Huntsman Hall 462, University of Pennsylvania, Philadelphia, PA 19104, USA. Email address: shepp@wharton.upenn.edu

**** Postal address: Wharton School, Department of Statistics, Huntsman Hall 447, University of Pennsylvania, Philadelphia, PA 19104, USA. Email address: steele@wharton.upenn.edu 
More recently, Houdré and Restrepo (2010) used purely probabilistic means to obtain a simpler proof of this result and the corresponding central limit theorem. Moreover, the methods of Houdré and Restrepo also apply to models of random words that are more refined than simple random selection from a set of permutations.

Here, we study the problem of making online selection of an alternating subsequence. That is, we now regard the sequence $x_{1}, x_{2}, \ldots$ as being presented to us sequentially, and, at the time $i$ when $x_{i}$ is presented, we must choose to include $x_{i}$ as a term of our subsequence-or we must reject $x_{i}$ as a member of the subsequence.

We will consider the sequence to be given by independent random variables $X_{1}, X_{2}, \ldots$ that have a common continuous distribution $F$, and, since we are only concerned with order properties, we can without loss of generality take the distribution to be uniform on $[0,1]$. We now need to be more explicit about the set $\Pi$ of feasible strategies for online selection. At time $i$, when presented with $X_{i}$, we must decide to select $X_{i}$ based on its value, the value of earlier members of the sequence, and the actions we have taken in the past. All of this information can be captured by saying that $\tau_{k}$, the index of the $k$ th selection, must be a stopping time with respect to the increasing sequence of $\sigma$-fields, $\mathcal{F}_{i}=\sigma\left\{X_{1}, X_{2}, \ldots, X_{i}\right\}, i=1,2, \ldots$ Given any feasible policy $\pi \in \Pi$, the random variable of interest here is $A_{n}^{o}(\pi)$, the number of selections made by the policy $\pi$ up to and including time $n$. In other words, $A_{n}^{o}(\pi)$ is equal to the largest $k$ for which there are stopping times $1 \leq \tau_{1}<\tau_{2}<\cdots<\tau_{k} \leq n$ such that $\left\{X_{\tau_{1}}, X_{\tau_{2}}, \ldots, X_{\tau_{k}}\right\}$ is an alternating sequence.

Theorem 1. (Asymptotic selection rate for large samples.) For each $n=1,2, \ldots$, there is a policy $\pi_{n}^{*} \in \Pi$ such that

$$
\mathrm{E}\left[A_{n}^{o}\left(\pi_{n}^{*}\right)\right]=\sup _{\pi \in \Pi} \mathrm{E}\left[A_{n}^{o}(\pi)\right],
$$

and, for such an optimal policy, we have, for all $n \geq 1$,

$$
(2-\sqrt{2}) n \leq \mathrm{E}\left[A_{n}^{o}\left(\pi_{n}^{*}\right)\right] \leq(2-\sqrt{2}) n+C,
$$

where $C$ is a constant with $C<11-4 \sqrt{2} \sim 5.343$. In particular, we have

$$
\mathrm{E}\left[A_{n}^{o}\left(\pi_{n}^{*}\right)\right] \sim(2-\sqrt{2}) n \text { as } n \rightarrow \infty .
$$

The proof of this result exploits the analysis of a closely related selection problem in which one considers a sample of size $N$, where $N$ is geometrically distributed with parameter $0<$ $\rho<1$ (so we have $\left.\mathrm{P}(N=k)=\rho^{k-1}(1-\rho), k=1,2,3, \ldots\right)$. Here we also assume that $N$ is independent of the sequence $X_{1}, X_{2}, \ldots$.

Parallel to our first theorem, we consider the number $A_{N}^{o}(\pi)$ of selections made by a feasible policy $\pi$ up to and including the random time $N$. The geometric smoothing provided by $N$ gives us a useful 'shift symmetry' that is missing in the fixed $n$ problem, and the analysis of a geometric sample turns out to be far more tractable. In particular, we can determine the exact expected length of the sequence selected by an optimal policy.

Theorem 2. (Expected selection size in geometric samples.) For each $0<\rho<1$, there is a $\pi^{*} \in \Pi$ such that

$$
\mathrm{E}\left[A_{N}^{o}\left(\pi^{*}\right)\right]=\sup _{\pi \in \Pi} \mathrm{E}\left[A_{N}^{o}(\pi)\right]
$$

and, for such an optimal policy, we have

$$
\mathrm{E}\left[A_{N}^{o}\left(\pi^{*}\right)\right]=\frac{3-2 \sqrt{2}-\rho+\rho \sqrt{2}}{\rho(1-\rho)} \sim(2-\sqrt{2})(1-\rho)^{-1} \text { as } \rho \rightarrow 1 .
$$


These theorems respectively tell us that optimal online selection yields subsequences that grow at a linear rate $(2-\sqrt{2}) n \sim 0.585 n$ or $(2-\sqrt{2}) \mathrm{E} N \sim 0.585 \mathrm{E} N$. This is about a $12 \%$ discount off the rate $\frac{2}{3} n \sim 0.667 n$ that we would obtain with a priori knowledge of the full finite sample $\left\{X_{1}, X_{2}, \ldots, X_{n}\right\}$, and this discount seems quite modest given the great difference in the knowledge that we have.

To build some intuition about these rates, we should also consider the 'maximally timid strategy' where we choose the first observation that falls in $[0,0.5]$, then the next observation that falls in $[0.5,1]$, then the next that falls in $[0,0.5]$, and so on. This strategy obviously leads to an asymptotic selection rate of $0.5 n$. Finally, we should also consider the 'purely greedy strategy' where we accept any new arrival that is feasible given the previous selections. Curiously enough, by a reasonably quick Markov chain calculation we can show that the greedy strategy leads to the same selection rate $0.5 n$ as that for the 'maximally timid strategy'.

We begin by proving Theorem 2, which will give us an exact formula for the expected number of selections made under the optimal policy for geometric samples. This result will then be used to prove the upper and lower bounds of Theorem 1 .

\section{Proof of Theorem 2}

Let $S_{i}$ denote the value of the last member of the subsequence selected up to and including time $i$. To keep track of the up-down nature of our selections, we then set $R_{i}=0$ if $S_{i}$ is a local minimum of $\left\{S_{0}, S_{1}, \ldots, S_{i}\right\}$ and $R_{i}=1$ if $S_{i}$ is a local maximum. To initialize our process, we set $S_{0}=1$ and $R_{0}=1$.

Next, we make the class $\Pi$ of feasible policies more explicit. For each $1 \leq i<\infty$ and each pair $\left(S_{i-1}, R_{i-1}\right)$, a feasible strategy $\pi$ specifies a set $\Delta_{i}\left(S_{i-1}, R_{i-1}\right)$ such that

$$
\Delta_{i}\left(S_{i-1}, 0\right) \subseteq\left[S_{i-1}, 1\right] \text { and } \Delta_{i}\left(S_{i-1}, 1\right) \subseteq\left[0, S_{i-1}\right],
$$

and $X_{i}$ is selected for our subsequence if and only if $X_{i} \in \Delta_{i}\left(S_{i-1}, R_{i-1}\right)$. For each $\pi \in \Pi$, we have the basic relation

$$
A_{N}^{o}(\pi)=\sum_{i=1}^{N} \mathbf{1}\left(X_{i} \in \Delta_{i}\left(S_{i-1}, R_{i-1}\right)\right)=\sum_{i=1}^{\infty} \mathbf{1}\left(X_{i} \in \Delta_{i}\left(S_{i-1}, R_{i-1}\right)\right) \mathbf{1}(i \leq N),
$$

and by taking expectations on both sides we have

$$
\mathrm{E}\left[A_{N}^{o}(\pi)\right]=\mathrm{E}\left[\sum_{i=1}^{\infty} \rho^{i-1} \mathbf{1}\left(X_{i} \in \Delta_{i}\left(S_{i-1}, R_{i-1}\right)\right)\right] .
$$

We came to this relation by considering random sample sizes with the geometric distribution, but the right-hand side of this identity can also be interpreted as the infinite-horizon discounted expected length of the alternating subsequence selected by $\pi$. We are interested in the policy $\pi^{*} \in \Pi$ such that

$$
\mathrm{E}\left[A_{N}^{o}\left(\pi^{*}\right)\right]=\sup _{\pi \in \Pi} \mathrm{E}\left[\sum_{i=1}^{\infty} \rho^{i-1} \mathbf{1}\left(X_{i} \in \Delta_{i}\left(S_{i-1}, R_{i-1}\right)\right)\right],
$$

and from the general theory of Markov decision problems we know that an optimal policy can be characterized as the solution of an associated Bellman equation. 


\subsection{First Bellman equation}

For any $i$ such that $S_{i}=s$ and $R_{i}=r$, we let $v(s, r)$ denote the expected number of selections made after time $i$ by an optimal policy. By the lack-of-memory property of the geometric distribution and the usual considerations of dynamic programming, we can now check that $v(s, r)$ satisfies the Bellman equation:

$$
v(s, r)= \begin{cases}\rho s v(s, 0)+\int_{s}^{1} \max \{\rho v(s, 0), 1+\rho v(x, 1)\} \mathrm{d} x & \text { if } r=0, \\ \rho(1-s) v(s, 1)+\int_{0}^{s} \max \{\rho v(s, 1), 1+\rho v(x, 0)\} \mathrm{d} x & \text { if } r=1 .\end{cases}
$$

To see why this equation holds, first consider the case when $r=0$ (so the next selection is to be a local maximum). With probability $\rho$ we get to see another observation $X_{i+1}$, and, with probability $s$, the value we observe is less than the previously selected value. In this case, we do not have the opportunity to make a selection, and this observation contributes the term $\rho s v(s, 0)$ to our equation.

Next, consider the case when $s<X_{i+1} \leq 1$. Now we can choose to select $X_{i+1}=x$ or not. If we do not select $X_{i+1}=x$, the expected number of subsequent selections is $\rho v(s, 0)$, and if we do select $X_{i+1}=x$, we increment the sequence by 1 and the expected number of subsequence selections that are made by an optimal policy in the future is given by $\rho v(x, 1)$. Since $X_{i+1}$ is uniformly distributed in [s,1], the expected optimal contribution is given by the second term of our Bellman equation (first line). The proof of the second line of the Bellman equation is completely analogous.

Finally, given a solution $v(s, r)$ to the Bellman equation (1), we have

$$
v(1,1)=\mathrm{E}\left[A_{N}^{o}\left(\pi^{*}\right)\right],
$$

so, now our goal is to determine $v(1,1)$. To do this, it will be useful to reorganize the Bellman equation (1) in a tidier form. This is possible since the solution $v(s, r)$ of the Bellman equation has a useful symmetry property.

Lemma 1. (Reflection identity.) For all $s \in[0,1]$, the solution $v(s, r)$ of the Bellman equation (1) satisfies

$$
v(s, 0)=v(1-s, 1) .
$$

Proof. The Bellman equation (1) is a fixed-point equation, and by the classical theory of dynamic programming, it can be solved by iteration (cf. Bertsekas and Shreve (1978, Section 9.5)). We will prove identity (2) by showing that it holds for the sequence of approximations; so it also holds for the limit.

We first set $v^{0}(s, r)=0$ for all $(s, r) \in[0,1] \times\{0,1\}$, and we note that $v^{0}$ trivially satisfies the reflection identity (2). Next, for our induction hypothesis, we assume that we have $v^{n-1}(s, 0)=v^{n-1}(1-s, 1)$ for all $s \in[0,1]$. The next iterate in the sequence is then given by

$$
v^{n}(s, 0)=\rho s v^{n-1}(s, 0)+\int_{s}^{1} \max \left\{\rho v^{n-1}(s, 0), 1+\rho v^{n-1}(x, 1)\right\} \mathrm{d} x .
$$

By applying our induction hypothesis on $v^{n-1}$ we then obtain

$$
v^{n}(s, 0)=\rho s v^{n-1}(1-s, 1)+\int_{s}^{1} \max \left\{\rho v^{n-1}(1-s, 1), 1+\rho v^{n-1}(1-x, 0)\right\} \mathrm{d} x .
$$


Now, after changing variables in the integral on the right-hand side, we find that

$$
\begin{aligned}
v^{n}(s, 0) & =\rho s v^{n-1}(1-s, 1)+\int_{0}^{1-s} \max \left\{\rho v^{n-1}(1-s, 1), 1+\rho v^{n-1}(x, 0)\right\} \mathrm{d} x \\
& =v^{n}(1-s, 1),
\end{aligned}
$$

and this completes the induction step. Now, for all $(s, r) \in[0,1] \times\{0,1\}$, we have $v^{n}(s, r) \rightarrow$ $v(s, r)$ as $n \rightarrow \infty$, so taking limits in the last identity completes the proof of the reflection identity.

\subsection{A simpler equation}

Using the reflection identity (2), we can put the Bellman equation (1) into a more graceful form. Specifically, if we introduce a single variable function $v(y)$ defined by setting

$$
v(y) \equiv v(y, 0)=v(1-y, 1),
$$

then substitution into our original equation (1) gives

$$
v(y)=\rho y v(y)+\int_{y}^{1} \max \{\rho v(y), 1+\rho v(1-x)\} \mathrm{d} x .
$$

Here we should note that, by the definition of $v(y)=v(y, 0), v(\cdot)$ is continuous, $v(1)=0$, and $v$ is nonincreasing on $[0,1]$. We will show shortly that $v$ is actually piecewise linear and it is constant on an initial segment of $[0,1]$.

\subsection{An alternative interpretation}

The symmetrized equation (3) can be used to obtain a new probabilistic interpretation of $v(y)$. To set this up, we first put

$$
f^{*}(y)=\inf \{x \in[y, 1]: \rho v(y) \leq 1+\rho v(1-x)\} .
$$

With this definition, we can rewrite (3) as

$$
v(y)=\rho f^{*}(y) v(y)+\int_{f^{*}(y)}^{1}\{1+\rho v(1-x)\} \mathrm{d} x .
$$

Thus, we remove the maximum from the integrand (3) at the price of introducing a threshold function $f^{*}$ that depends on $v$.

We now recursively define random variables $\left\{Y_{i}: i=1,2, \ldots\right\}$ by setting $Y_{0}=0$ and taking

$$
Y_{i}= \begin{cases}Y_{i-1} & \text { if } X_{i}<f^{*}\left(Y_{i-1}\right), \\ 1-X_{i} & \text { if } X_{i} \geq f^{*}\left(Y_{i-1}\right),\end{cases}
$$

and finally introduce a new value function

$$
v_{0}(y) \equiv \mathrm{E}\left[\sum_{i=1}^{\infty} \rho^{i-1} \mathbf{1}\left(X_{i} \geq f^{*}\left(Y_{i-1}\right)\right) \mid Y_{0}=y\right]
$$

The next proposition shows that $v_{0}(y)$ is actually equal to $v(y)$. Furthermore, we obtain a concrete characterization of the threshold function $f^{*}$. 
Proposition 1. (Structure of the solution of the Bellman equation.) We have the following characterizations of $f^{*}$ and $v_{0}$.

(i) There is a unique $\xi_{0} \in[0,1]$ such that

$$
f^{*}(y)=\max \left\{\xi_{0}, y\right\} \quad \text { for all } 0 \leq y \leq 1,
$$

and, moreover, $0 \leq \xi_{0}<\frac{1}{2}$.

(ii) The function $v_{0}(\cdot)$ is a solution of the Bellman equation (3), so, by uniqueness, we have $v_{0}(y)=v(y)$ for all $0 \leq y \leq 1$.

Proof. From the definition of $f^{*}$ we see that

$$
\rho v(y) \leq 1+\rho v(1-y) \quad \Longrightarrow \quad f^{*}(y)=y .
$$

Now, for $\frac{1}{2} \leq y$, we have $1-y \leq y$, so the monotonicity of $v$ gives us the bound $\rho v(y) \leq$ $1+\rho v(1-y)$; consequently, we have $f^{*}(y)=y$ for $y \in\left[\frac{1}{2}, 1\right]$.

If condition (7) holds for all $y \in\left[0, \frac{1}{2}\right)$ then $f^{*}(y)=y$ for all $y \in[0,1]$ and we can take $\xi_{0}=0$. Otherwise, there is a $y_{0} \in\left[0, \frac{1}{2}\right)$ for which we have

$$
1+\rho v\left(1-y_{0}\right)<\rho v\left(y_{0}\right) .
$$

For $\Delta(y)=1+\rho v(1-y)-\rho v(y)$, we then have $\Delta\left(y_{0}\right)<0$ and $\Delta(1)=1+\rho v(0)>0$, so by continuity we have $S=\{y: \Delta(y)=0\} \neq \varnothing$. If we now take $\xi_{0}$ to be the infimum of $S$ then $\xi_{0} \in\left[y_{0}, \frac{1}{2}\right) \subset\left[0, \frac{1}{2}\right)$ and $\rho v\left(\xi_{0}\right)=1+\rho v\left(1-\xi_{0}\right)$. The definition of $f^{*}$ now tells us that $f^{*}(y)=\xi_{0}$ for $y \leq \xi_{0}$ and $f^{*}(y)=y$ for $\xi_{0} \leq y$. This completes the proof of the first part of the proposition.

Finally, to check that $v_{0}$ solves (6), we just condition on the value of $X_{1}$ and calculate the expectation of the sum. When we take the total expectation, we obtain the right-hand side of (5).

\subsection{Characterization of the critical value}

Now that we know that the threshold function $f^{*}$ for the solution of the Bellman equation (3) has the form $f^{*}(y)=\max \left\{\xi_{0}, y\right\}$ for some $\xi_{0} \in\left[0, \frac{1}{2}\right)$, the main problem is to find $\xi_{0}$. The natural plan is to fix $\xi \in\left[0, \frac{1}{2}\right]$ and to consider a general selection function of the form $f(y)=\max \{\xi, y\} \equiv(\xi \vee y)$. We then want to calculate the associated value function and to optimize over $\xi$.

The associated value function is given by

$$
V(y, \xi, \rho)=\mathrm{E}\left[\sum_{i=1}^{\infty} \rho^{i-1} \mathbf{1}\left(X_{i} \geq \max \left\{\xi, Y_{i-1}\right\}\right) \mid Y_{0}=y\right],
$$

and Proposition 1 then tells us that

$$
\max _{\xi \in[0,1 / 2]} V(y, \xi, \rho)=v(y) \text { for all } y \in[0,1] .
$$

If we use the abbreviated notation $V(y) \equiv V(y, \xi, \rho)$ then by conditioning on $X_{1}$ in (8) we see that $V(y)$ satisfies the integral equation

$$
\begin{aligned}
V(y) & =(\xi \vee y) \rho V(y)+\int_{\xi \vee y}^{1}\{1+\rho V(1-x)\} \mathrm{d} x \\
& =(\xi \vee y) \rho V(y)+\int_{0}^{1-(\xi \vee y)}\{1+\rho V(x)\} \mathrm{d} x .
\end{aligned}
$$


This equation has several attractive features. In particular, if we set $y=1$ then from $0<\rho<1$ we see that $V(1)=0$. Also, by writing

$$
V(y)=\frac{1}{1-\rho(\xi \vee y)} \int_{0}^{1-(\xi \vee y)}\{1+\rho V(x)\} \mathrm{d} x,
$$

we see that the right-hand side does not change when $y \in[0, \xi]$, so we have

$$
V(y)=V\left(y^{\prime}\right) \text { for all } 0 \leq y, y^{\prime} \leq \xi .
$$

From now on, we will let $V^{\prime}(\xi)$ denote the right derivative of the integral equation (9) evaluated at $\xi$, and $V^{\prime}(1-\xi)$ denote the left derivative of (9) evaluated at $1-\xi$. Elsewhere, $V^{\prime}(y)$ simply denotes the derivative of (9) evaluated at $y$.

Lemma 2. The solution of (9) satisfies the following four conditions:

(i) $V(1-\xi)(1-\rho+\rho \xi)=\xi+\rho \xi V(\xi)$;

(ii) $V^{\prime}(\xi)(1-\rho \xi)=\rho[V(\xi)-V(1-\xi)]-1$;

(iii) $V^{\prime}(1-\xi)(1-\rho+\rho \xi)=\rho[V(1-\xi)-V(\xi)]-1$;

(iv) $V^{\prime}(1-\xi)(1-\rho+\rho \xi)^{2}(1-\rho \xi)=V^{\prime}(\xi)(1-\rho \xi)^{2}(1-\rho+\rho \xi)+(1-\rho+\rho \xi)^{2}-(1-\rho \xi)^{2}$.

Proof. Conditions (i)-(iii) are easy to check. Condition (i) is just (9) evaluated at $1-\xi$ together with (10). Conditions (ii) and (iii) simply follow by evaluating (9) at $\xi$ and $1-\xi$, respectively, and by differentiating both sides with respect to $\xi$.

The proof of condition (iv) requires more work. Consider $y \in(\xi, 1-\xi)$ so that the integral equation (9) becomes

$$
V(y)=y \rho V(y)+\int_{0}^{1-y}\{1+\rho V(x)\} \mathrm{d} x .
$$

Differentiating once we have

$$
V^{\prime}(y)(1-\rho y)=\rho[V(y)-V(1-y)]-1,
$$

and differentiating again gives

$$
V^{\prime \prime}(y)(1-\rho y)-\rho V^{\prime}(y)=\rho V^{\prime}(y)+\rho V^{\prime}(1-y) .
$$

To estimate the value of $V^{\prime}(1-y)$, we note that $1-y \in(\xi, 1-\xi)$, and we evaluate the integral equation (9) at $1-y$. We then differentiate with respect to $y$ to obtain

$$
V^{\prime}(1-y)(1-\rho+\rho y)=\rho[V(1-y)-V(y)]-1 .
$$

By combining (11) and (13), we then have

$$
V^{\prime}(1-y)=(1-\rho+\rho y)^{-1}\left(-V^{\prime}(y)(1-\rho y)-2\right),
$$

which we can substitute into the last addend of (12) to obtain

$$
V^{\prime \prime}(y)(1-\rho y)(1-\rho+\rho y)=V^{\prime}(y) \rho(1-2 \rho+3 \rho y)-2 \rho .
$$


By multiplying both sides of $(14)$ by $(1-\rho y)$ we obtain the critical identity

$$
V^{\prime \prime}(y)(1-\rho y)^{2}(1-\rho+\rho y)=V^{\prime}(y) \rho(1-\rho y)(1-2 \rho+3 \rho y)-2 \rho(1-\rho y) .
$$

For $h(y)=(1-\rho y)^{2}(1-\rho+\rho y)$, note that $h^{\prime}(y)=-\rho(1-\rho y)(1-2 \rho+3 \rho y)$, so that we can rewrite identity (15) as

$$
V^{\prime \prime}(y) h(y)+V^{\prime}(y) h^{\prime}(y)-\left[(1-\rho y)^{2}\right]^{\prime}=0 .
$$

An immediate integration then gives

$$
V^{\prime}(y) h(y)-(1-\rho y)^{2}=C,
$$

where $C$ is a constant, and if we take $C=V^{\prime}(\xi) h(\xi)-(1-\rho \xi)^{2}$, we find that

$$
V^{\prime}(y)=V^{\prime}(\xi) \frac{h(\xi)}{h(y)}+\frac{(1-\rho y)^{2}-(1-\rho \xi)^{2}}{h(y)} \text { for all } \xi<y<1-\xi .
$$

Finally, on setting $y=1-\xi$ we recover the desired condition (iv).

\subsection{Calculation of the critical value}

Conditions (i)-(iv) of Lemma 2 generate a system of four equations in four unknowns, $V(\xi), V(1-\xi), V^{\prime}(\xi)$, and $V^{\prime}(1-\xi)$. By solving this system we find that

$$
\begin{aligned}
V(\xi) & =\frac{2-2 \xi-\rho+2 \rho \xi-2 \rho \xi^{2}}{2(1-\rho)(1-\rho \xi)}, \\
V(1-\xi) & =\frac{\rho\left(2-4 \rho \xi-\rho^{2}+4 \rho^{2} \xi-2 \rho^{2} \xi^{2}\right)}{2(1-\rho)(1-\rho \xi)^{2}(1-\rho+\rho \xi)}, \\
V^{\prime}(\xi) & =\frac{-2+4 \rho-4 \rho \xi-\rho^{2}+2 \rho^{2} \xi^{2}}{2(1-\rho \xi)^{2}(1-\rho+\rho \xi)}, \\
V^{\prime}(1-\xi) & =\frac{-2+4 \rho \xi+\rho^{2}-4 \rho^{2} \xi+2 \rho^{2} \xi^{2}}{2(1-\rho \xi)(1-\rho+\rho \xi)^{2}} .
\end{aligned}
$$

Finally, by substituting (18) into (16) we obtain

$$
V^{\prime}(y)=\frac{-(2-\rho)^{2}+2(1-\rho y)^{2}}{2(1-\rho+\rho y)(1-\rho y)^{2}} \quad \text { for all } \xi<y<1-\xi .
$$

Now, given any $\xi$, we want to compute $V(0, \xi, \rho)$. We first recall that we have $V(1, \xi, \rho)=0$ and $V(y, \xi, \rho)=V(\xi, \xi, \rho)$ for all $0 \leq y \leq \xi$. We therefore find that $\partial V(y, \xi, \rho) / \partial y=0$ on $0 \leq y \leq \xi$, so on integrating we have

$$
V(1, \xi, \rho)-V(0, \xi, \rho)=\int_{0}^{1} V^{\prime}(y) \mathrm{d} y=\int_{\xi}^{1} V^{\prime}(y) \mathrm{d} y,
$$

and, hence,

$$
V(0, \xi, \rho)=-\int_{\xi}^{1} V^{\prime}(y) \mathrm{d} y
$$


We now optimize this last quantity with respect to $\xi$. By differentiating both sides with respect to $\xi$ we obtain

$$
\frac{\partial}{\partial \xi} V(0, \xi, \rho)=V^{\prime}(\xi),
$$

and we are interested in the value $\xi_{0}$ such that

$$
V^{\prime}\left(\xi_{0}\right)=0
$$

Formula (18) for $V^{\prime}\left(\xi_{0}\right)$ tells us that $V^{\prime}\left(\xi_{0}\right)=0$ if and only if

$$
2\left(1-\rho \xi_{0}\right)^{2}=(2-\rho)^{2} .
$$

We therefore find that the unique choice for $\xi_{0}$ is given by

$$
\xi_{0}=\frac{1}{\sqrt{2}}+\frac{1-\sqrt{2}}{\rho} .
$$

A routine calculation verifies that $V^{\prime \prime}\left(\xi_{0}\right)<0$, so we have found our maximum.

When we evaluate $V\left(\xi_{0}, \xi_{0}, \rho\right)$ using (17), we find that

$$
V\left(\xi_{0}, \xi_{0}, \rho\right)=\frac{3-2 \sqrt{2}-\rho+\rho \sqrt{2}}{\rho(1-\rho)},
$$

and this gives us the main formula, of Theorem 2. From this formula, it is immediate that

$$
\lim _{\rho \uparrow 1}(1-\rho) V\left(\xi_{0}, \xi_{0}, \rho\right)=2-\sqrt{2},
$$

completing the proof of Theorem 2 .

\section{Proof of Theorem 1 from Theorem 2}

We will use our results for geometric sample sizes to obtain both lower and upper bounds for the finite-sample size selection problem. The lower bound is the easiest. For fixed $n$, we can use the (now suboptimal) policy from an appropriately chosen geometric sample size problem. The proof of the upper bound is considerably harder, and the method will be described later in this section. Before making these arguments, we need to organize a few structural observations.

\subsection{Selection policies and a Bellman equation for finite samples}

When the sample size $n$ is deterministic and known, the feasible policies need to take this information into account. In particular, the selection thresholds will no longer be stationary; they will depend on the number of sample elements that remain to be seen.

As in Section 2, we consider the pairs $\left(S_{i-1}, R_{i-1}\right), 1 \leq i \leq n$, where $S_{i-1}$ is the size of the last selection made before time $i$ and $R_{i-1}$ is 0 or 1 according to whether the last selection was a local minimum or a local maximum. A feasible policy $\pi \in \Pi$ again specifies a set $\Delta_{i, n}\left(S_{i-1}, R_{i-1}\right)$ that depends only on past actions, but now we have dependence on the decision time $i$ and on the sample size $n$. For any policy $\pi \in \Pi$, the expected size of the selected sample can then be written as

$$
\mathrm{E}\left[A_{n}^{o}(\pi)\right]=\mathrm{E}\left[\sum_{i=1}^{n} \mathbf{1}\left(X_{i} \in \Delta_{i, n}\left(S_{i-1}, R_{i-1}\right)\right)\right],
$$


and there is an optimal policy $\pi_{n}^{*}$ for which we have

$$
\mathrm{E}\left[A_{n}^{o}\left(\pi_{n}^{*}\right)\right]=\sup _{\pi \in \Pi} \mathrm{E}\left[A_{n}^{o}(\pi)\right] .
$$

In this case, an optimal policy can be characterized as the solution to a finite-sample Bellman equation. Specifically, for $1 \leq i \leq n$, we have

$$
v_{i, n}(s, r)= \begin{cases}s v_{i+1, n}(s, 0)+\int_{s}^{1} \max \left\{v_{i+1, n}(s, 0), 1+v_{i+1, n}(x, 1)\right\} \mathrm{d} x & \text { if } r=0, \\ (1-s) v_{i+1, n}(s, 1)+\int_{0}^{s} \max \left\{v_{i+1, n}(s, 1), 1+v_{i+1, n}(x, 0)\right\} \mathrm{d} x & \text { if } r=1,\end{cases}
$$

and the backward induction begins by setting $v_{n+1, n}(s, r) \equiv 0$ for all $(s, r)$ in $[0,1] \times\{0,1\}$. This equation is justified by the same considerations that were used in the derivation of (1).

\subsection{Symmetry and simplification}

For the finite-sample size problem, we lose much of the nice symmetry of the geometric sample size problem. Nevertheless, the solution of the finite-sample Bellman equation still has a reflection identity analogous to that given by Lemma 1.

Lemma 3. The solution of the finite-sample Bellman equation satisfies

$$
v_{i, n}(s, 0)=v_{i, n}(1-s, 1) \quad \text { for all } 1 \leq i \leq n \text { and all } s \in[0,1] .
$$

Proof. Again, we use an induction argument, but this time we do not need to take limits of an infinite sequence of approximate solutions. Instead, we simply use backward induction and always work with exact solutions.

Since we have $v_{n, n}(s, 0)=1-s$ and $v_{n, n}(1-s, 1)=1-s$, we see that (20) holds for $i=n$, so we suppose by induction that $v_{i+1, n}(s, 0)=v_{i+1, n}(1-s, 1)$. We then have

$$
v_{i, n}(s, 0)=s v_{i+1, n}(s, 0)+\int_{s}^{1} \max \left\{v_{i+1, n}(s, 0), 1+v_{i+1, n}(x, 1)\right\} \mathrm{d} x,
$$

so by applying the induction hypothesis on the right-hand side we obtain

$$
v_{i, n}(s, 0)=s v_{i+1, n}(1-s, 1)+\int_{s}^{1} \max \left\{v_{i+1, n}(1-s, 1), 1+v_{i+1, n}(1-x, 0)\right\} \mathrm{d} x .
$$

If we now change the variable in this last integral, we obtain

$$
\begin{aligned}
v_{i, n}(s, 0) & =s v_{i+1, n}(1-s, 1)+\int_{0}^{1-s} \max \left\{v_{i+1, n}(1-s, 1), 1+v_{i+1, n}(x, 0)\right\} \mathrm{d} x \\
& =v_{i, n}(1-s, 1),
\end{aligned}
$$

and this completes the induction step.

We can now define a new single variable function $v_{i, n}(y)$ by setting

$$
v_{i, n}(y)=v_{i, n}(y, 0)=v_{i, n}(1-y, 1),
$$

and by substitution into the original finite-sample Bellman equation we have

$$
v_{i, n}(y)=y v_{i+1, n}(y)+\int_{y}^{1} \max \left\{v_{i+1, n}(y), 1+v_{i+1, n}(1-x)\right\} \mathrm{d} x .
$$

Here we should also note that $v_{i, n}(\cdot)$ is continuous and nonincreasing on $[0,1]$ for all $1 \leq i \leq n$. 


\subsection{The threshold functions}

We now define the finite-sample equivalent of the threshold function (4) by setting

$$
f_{i, n}^{*}(y)=\inf \left\{x \in[y, 1]: v_{i+1, n}(y) \leq 1+v_{i+1, n}(1-x)\right\}
$$

If we then set $Y_{0}=0$ and define $Y_{i}$ recursively by setting

$$
Y_{i}= \begin{cases}Y_{i-1} & \text { if } X_{i}<f_{i, n}^{*}\left(Y_{i-1}\right) \\ 1-X_{i} & \text { if } X_{i} \geq f_{i, n}^{*}\left(Y_{i-1}\right)\end{cases}
$$

then, in complete parallel to the geometric case, we see that the solution of the finite-sample Bellman equation (22) can be written more probabilistically as

$$
v_{1, n}(y)=\mathrm{E}\left[\sum_{i=1}^{n} \mathbf{1}\left(X_{i} \geq f_{i, n}^{*}\left(Y_{i-1}\right)\right) \mid Y_{0}=y\right] .
$$

Finally, from (21) we have

$$
v_{1, n}(0)=v_{1, n}(0,0)=v_{1, n}(1,1)=\mathrm{E}\left[A_{n}^{o}\left(\pi_{n}^{*}\right)\right],
$$

and this gives us the last piece of structural information that we need.

\subsection{Proof of the lower bound}

To prove that

$$
(2-\sqrt{2}) n \leq \mathrm{E}\left[A_{n}^{o}\left(\pi_{n}^{*}\right)\right] \text { for all } n \geq 1,
$$

we only need to choose a good suboptimal policy. We now fix $\xi \in\left[0, \frac{1}{2}\right]$, and we consider the policy in which $X_{i}$ is selected if and only if $X_{i} \geq \max \left\{\xi, Y_{i-1}\right\}$. Here, $Y_{0}=y$ is in the interval $[0,1-\xi]$ and the $Y_{i}$ s are defined recursively by setting

$$
Y_{i}= \begin{cases}Y_{i-1} & \text { if } X_{i}<\max \left\{\xi, Y_{i-1}\right\} \\ 1-X_{i} & \text { if } X_{i} \geq \max \left\{\xi, Y_{i-1}\right\}\end{cases}
$$

The sequence $\left\{Y_{i}: i=0,1, \ldots\right\}$ is a discrete-time Markov chain on the state space $[0,1-\xi]$. For a measurable $A \subseteq[0,1-\xi]$, we let $|A|$ denote the Lebesgue measure of $A$, and, for a measurable set $B \subseteq[0,1-\xi]$, we write $1-B$ as shorthand for the set $\{u \in[0,1]: 1-u \in B\}$. Given these abbreviations, the transition kernel of the process $\left\{Y_{i}: i=0,1, \ldots\right\}$ can be written as

$$
K(x, C)=\mathbf{1}(x \in C)(\xi \vee x)+|1-C \cap[\xi \vee x, 1]| .
$$

It is now easy to check that the process $\left\{Y_{i}\right\}$ has a unique stationary distribution $\gamma$, and, in fact, $\gamma$ is just the uniform distribution on $[0,1-\xi]$, (i.e. $\gamma(C)=(1-\xi)^{-1}|C|$ for all measurable $C \subseteq[0,1-\xi])$.

For any starting value $Y_{0}=y \in[0,1-\xi]$, the suboptimality of the selection functions $\max \left\{\xi, Y_{i-1}\right\}$ gives

$$
\mathrm{E}\left[\sum_{i=1}^{n} \mathbf{1}\left(X_{i} \geq \max \left\{\xi, Y_{i-1}\right\}\right) \mid Y_{0}=y\right] \leq v_{1, n}(y) .
$$


Since $v_{1, n}(y)$ is nonincreasing in $y$, we see that, for any starting distribution $\mu$ supported on $[0,1-\xi]$, we have

$$
\mathrm{E}_{\mu}\left[\sum_{i=1}^{n} \mathbf{1}\left(X_{i} \geq \max \left\{\xi, Y_{i-1}\right\}\right)\right] \leq \mathrm{E}_{\mu}\left[v_{1, n}\left(Y_{0}\right)\right] \leq v_{1, n}(0)=\mathrm{E}\left[A_{n}^{o}\left(\pi_{n}^{*}\right)\right] .
$$

If we choose the starting distribution $\mu$ to be the stationary distribution $\gamma$ then

$$
\mathrm{E}_{\gamma}\left[\sum_{i=1}^{n} \mathbf{1}\left(X_{i} \geq \max \left\{\xi, Y_{i-1}\right\}\right)\right]=n \mathrm{E}_{\gamma}\left[1-\max \left\{\xi, Y_{0}\right\}\right] \leq \mathrm{E}\left[A_{n}^{o}\left(\pi_{n}^{*}\right)\right]
$$

and we can compute the first expression explicitly. So, we have

$$
\mathrm{E}_{\gamma}\left[1-\max \left\{\xi, Y_{0}\right\}\right]=\frac{1}{1-\xi} \int_{0}^{1-\xi} 1-\max \{\xi, y\} \mathrm{d} y=\frac{1-2 \xi^{2}}{2(1-\xi)} .
$$

We can maximize this by taking $\xi=1-2^{-1 / 2}$ (as in (19) when $\rho=1$ ), and we then obtain

$$
\mathrm{E}_{\gamma}\left[1-\max \left\{\xi, Y_{0}\right\}\right]=2-\sqrt{2} .
$$

Together with inequality (26), this completes the proof of our lower bound.

\subsection{Proof of the upper bound}

The proof of the upper bound in Theorem 1 requires a more sustained argument. Unlike the problem for geometric samples, the value function $v_{i, n}(\cdot)$ is no longer constant on an initial segment of $[0,1]$. Nevertheless, the next proposition tells us that the value function does have a useful uniform boundedness on an initial segment. This is the first of several structural observations that we will need to obtain our upper bound for $\mathrm{E}\left[A_{n}^{o}\left(\pi_{n}^{*}\right)\right]$.

Proposition 2. (Value function initial segment bounds.) For all $0 \leq u<\frac{1}{6}$ and $n \geq 2$, the functions $v_{i, n}(\cdot)$ defined by the Bellman recursion (22) satisfy

(i) $1<v_{i, n}(u)-v_{i, n}\left(\frac{5}{6}\right)$ for all $1 \leq i \leq n-1$;

(ii) $v_{i, n}(u)-v_{i, n}\left(\frac{1}{6}\right)<1$ for all $1 \leq i \leq n$.

Moreover, for $n \geq 3$, the threshold functions $f_{i, n}^{*}(y)$ defined in (23) are guaranteed to satisfy $\frac{1}{6} \leq f_{i, n}^{*}(y)$ for $y \in[0,1]$ and $1 \leq i \leq n-2$.

Naturally enough, the proof of this proposition depends on inductive arguments that exploit the defining Bellman equation. The first of these arguments gives us some control over the changes of $v_{i, n}(u)$ when we change both $i$ and $u$.

Lemma 4. (Restricted supermodularity.) For $y \in\left[0, \frac{1}{2}\right]$ and $u \in[y, 1-y]$, the functions $\left\{v_{i, n}(\cdot)\right\}$ defined by the Bellman recursion (22) satisfy

$$
v_{i+1, n}(u)-v_{i+1, n}(1-y) \leq v_{i, n}(u)-v_{i, n}(1-y) \quad \text { for all } 1 \leq i \leq n .
$$

Proof. We use backward induction on $i$, and, since $n$ is fixed, we abbreviate $v_{i, n}(\cdot)$ by $v_{i}(\cdot)$. For $i=n$, we have $v_{n+1}(u)=0$ for all $u \in[0,1]$. Moreover, $v_{n}(u)=1-u$ and $v_{n}(1-y)=y$, so we have

$$
v_{n+1}(u)-v_{n+1}(1-y) \leq v_{n}(u)-v_{n}(1-y) \text { for all } u \in[y, 1-y] .
$$


Now, for our backward induction, we can assume more generally that

$$
v_{i+1}(u)-v_{i+1}(1-y) \leq v_{i}(u)-v_{i}(1-y) \text { for all } u \in[y, 1-y] .
$$

The Bellman equation (22) then gives

$$
\begin{aligned}
v_{i-1}(u)-v_{i-1}(1-y)= & u v_{i}(u)+\int_{u}^{1} \max \left\{v_{i}(u), 1+v_{i}(1-x)\right\} \mathrm{d} x \\
& -(1-y) v_{i}(1-y)-\int_{1-y}^{1} \max \left\{v_{i}(1-y), 1+v_{i}(1-x)\right\} \mathrm{d} x,
\end{aligned}
$$

and, since $u \leq 1-y$, we can break up the first integral to obtain

$$
\begin{aligned}
v_{i-1}(u) & -v_{i-1}(1-y) \\
= & u v_{i}(u)-(1-y) v_{i}(1-y)+\int_{u}^{1-y} \max \left\{v_{i}(u), 1+v_{i}(1-x)\right\} \mathrm{d} x \\
& +\int_{1-y}^{1}\left(\max \left\{v_{i}(u), 1+v_{i}(1-x)\right\}-\max \left\{v_{i}(1-y), 1+v_{i}(1-x)\right\}\right) \mathrm{d} x .
\end{aligned}
$$

For $x \in[1-y, 1]$, we have $v_{i}(y) \leq v_{i}(1-x)$ since $v_{i}(\cdot)$ is nonincreasing on $[0,1]$. Therefore, since $y \leq u \leq 1-y$, we have $v_{i}(1-y) \leq v_{i}(u) \leq v_{i}(y)$, so that, for $x \in[1-y, 1]$, we have

$$
\max \left\{v_{i}(u), 1+v_{i}(1-x)\right\}=\max \left\{v_{i}(1-y), 1+v_{i}(1-x)\right\}=1+v_{i}(1-x),
$$

and we see that integral (27) equals 0 . We now have just the identity

$$
v_{i-1}(u)-v_{i-1}(1-y)=u v_{i}(u)-(1-y) v_{i}(1-y)+\int_{u}^{1-y} \max \left\{v_{i}(u), 1+v_{i}(1-x)\right\} \mathrm{d} x,
$$

or, equivalently,

$$
\begin{aligned}
v_{i-1}(u)-v_{i-1}(1-y)= & u\left(v_{i}(u)-v_{i}(1-y)\right) \\
& +\int_{u}^{1-y} \max \left\{v_{i}(u)-v_{i}(1-y), 1+v_{i}(1-x)-v_{i}(1-y)\right\} \mathrm{d} x .
\end{aligned}
$$

Changing variables in this last integral then gives the convenient identity

$$
\begin{aligned}
v_{i-1}(u)-v_{i-1}(1-y)= & u\left(v_{i}(u)-v_{i}(1-y)\right) \\
& +\int_{y}^{1-u} \max \left\{v_{i}(u)-v_{i}(1-y), 1+v_{i}(x)-v_{i}(1-y)\right\} \mathrm{d} x .
\end{aligned}
$$

Since $y \leq u$ and $1-u \leq 1-y$, we can now use our induction assumption to obtain

$$
\begin{aligned}
& v_{i-1}(u)-v_{i-1}(1-y) \\
& \geq u\left(v_{i+1}(u)-v_{i+1}(1-y)\right) \\
& \quad+\int_{y}^{1-u} \max \left\{v_{i+1}(u)-v_{i+1}(1-y), 1+v_{i+1}(x)-v_{i+1}(1-y)\right\} \mathrm{d} x \\
& =v_{i}(u)-v_{i}(1-y),
\end{aligned}
$$

where the last equality follows from recursion (28). 
We can now complete the proof of the value function bounds in Proposition 2.

Proof of Proposition 2. We begin by proving (i) by backwards induction on $i$. As before, since $n \geq 2$ is fixed, we abbreviate $v_{i, n}(\cdot)$ by $v_{i}(\cdot)$. For $i=n-1$, one iteration of the recursive definition of the Bellman equation (22) gives $v_{n-1}(x)=\frac{3}{2}\left(1-x^{2}\right)$, so $v_{n-1}(u)-v_{n-1}\left(\frac{5}{6}\right)=$ $\frac{3}{2}\left(\frac{25}{36}-u^{2}\right)>1$ since by hypothesis we have $u<\frac{1}{6}$. We now make the induction assumption

$$
1<v_{i+1}(u)-v_{i+1}\left(\frac{5}{6}\right) \quad \text { for } 0 \leq u<\frac{1}{6},
$$

and observe from the Bellman equation (22) that

$$
\begin{aligned}
v_{i}(u)-v_{i}\left(\frac{5}{6}\right)= & u v_{i+1}(u)+\int_{u}^{1} \max \left\{v_{i+1}(u), 1+v_{i+1}(1-x)\right\} \mathrm{d} x \\
& -\frac{5}{6} v_{i+1}\left(\frac{5}{6}\right)-\int_{5 / 6}^{1} \max \left\{v_{i+1}\left(\frac{5}{6}\right), 1+v_{i+1}(1-x)\right\} \mathrm{d} x .
\end{aligned}
$$

Since $u<\frac{1}{6}$, the monotonicity of $v_{i}(\cdot)$ implies that $v_{i+1}\left(\frac{5}{6}\right) \leq v_{i+1}(u)$. So, for $x \in\left[\frac{5}{6}, 1\right]$, we have $\max \left\{v_{i+1}\left(\frac{5}{6}\right), 1+v_{i+1}(1-x)\right\} \leq \max \left\{v_{i+1}(u), 1+v_{i+1}(1-x)\right\}$. This gives us the lower bound

$$
\begin{aligned}
& u\left(v_{i+1}(u)-v_{i+1}\left(\frac{5}{6}\right)\right)+\int_{u}^{5 / 6} \max \left\{v_{i+1}(u)-v_{i+1}\left(\frac{5}{6}\right), 1+v_{i+1}(1-x)-v_{i+1}\left(\frac{5}{6}\right)\right\} \mathrm{d} x \\
& \quad \leq v_{i}(u)-v_{i}\left(\frac{5}{6}\right) .
\end{aligned}
$$

To get a lower bound for the integral of the maximum, we replace the integrand by $v_{i+1}(u)-$ $v_{i+1}\left(\frac{5}{6}\right)$ on $\left[u, \frac{1}{6}\right)$ and replace it by $1+v_{i+1}(1-x)-v_{i+1}\left(\frac{5}{6}\right)$ on $\left[\frac{1}{6}, \frac{5}{6}\right]$. Changing variables then gives

$$
\frac{1}{6}\left(v_{i+1}(u)-v_{i+1}\left(\frac{5}{6}\right)\right)+\int_{1 / 6}^{5 / 6}\left\{1+v_{i+1}(x)-v_{i+1}\left(\frac{5}{6}\right)\right\} \mathrm{d} x \leq v_{i}(u)-v_{i}\left(\frac{5}{6}\right) .
$$

By our induction hypothesis, the first addend satisfies the bound

$$
\frac{1}{6}<\frac{1}{6}\left(v_{i+1}(u)-v_{i+1}\left(\frac{5}{6}\right)\right),
$$

and, by Lemma 4, the second integral satisfies the bound

$$
\int_{1 / 6}^{5 / 6}\left\{1+v_{n}(x)-v_{n}\left(\frac{5}{6}\right)\right\} \mathrm{d} x \leq \int_{1 / 6}^{5 / 6}\left\{1+v_{i+1}(x)-v_{i+1}\left(\frac{5}{6}\right)\right\} \mathrm{d} x .
$$

If we now recall that $v_{n}(x)=1-x$ and compute the integral on the left-hand side, we then obtain

$$
\frac{32}{36} \leq \int_{1 / 6}^{5 / 6}\left\{1+v_{i+1}(x)-v_{i+1}\left(\frac{5}{6}\right)\right\} \mathrm{d} x .
$$

Finally, adding (30) and (31) and recalling (29) gives us our target bound

$$
1<\frac{38}{36} \leq v_{i}(u)-v_{i}\left(\frac{5}{6}\right) \text {. }
$$

To prove condition (ii), we again use backwards induction. For $i=n$, we have $v_{n}(u)=1-u$, so $v_{n}(u)-v_{n}\left(\frac{1}{6}\right)=\frac{1}{6}-u<1$. Suppose now that

$$
v_{i+1}(u)-v_{i+1}\left(\frac{1}{6}\right)<1 \quad \text { for } 0 \leq u<\frac{1}{6} \text {. }
$$


The Bellman recursion (22) then gives

$$
\begin{aligned}
v_{i}(u)- & v_{i}\left(\frac{1}{6}\right) \\
\leq & \int_{0}^{1 / 6} \max \left\{v_{i+1}(u)-v_{i+1}\left(\frac{1}{6}\right), 1+v_{i+1}(1-x)-v_{i+1}\left(\frac{1}{6}\right)\right\} \mathrm{d} x \\
& +\int_{1 / 6}^{5 / 6}\left(\max \left\{v_{i+1}(u), 1+v_{i+1}(x)\right\}-\max \left\{v_{i+1}\left(\frac{1}{6}\right), 1+v_{i+1}(x)\right\}\right) \mathrm{d} x \\
& +\int_{5 / 6}^{1}\left(\max \left\{v_{i+1}(u), 1+v_{i+1}(1-x)\right\}-\max \left\{v_{i+1}\left(\frac{1}{6}\right), 1+v_{i+1}(1-x)\right\}\right) \mathrm{d} x .
\end{aligned}
$$

For $x \in\left[0, \frac{1}{6}\right]$, we can check that the first integrand is bounded by 1 . To see this, we first note that the left maximand is bounded by 1 by the induction assumption. Next, we note that $v_{i+1}(1-x) \leq v_{i+1}\left(\frac{5}{6}\right)$ so, for the second maximand, we have the bound $1+v_{i+1}(1-x)-$ $v_{i+1}\left(\frac{1}{6}\right) \leq 1+v_{i+1}\left(\frac{5}{6}\right)-v_{i+1}\left(\frac{1}{6}\right)$ and this last term is nonpositive by inequality (i).

For $x \in\left[\frac{1}{6}, \frac{5}{6}\right]$, the second integrand is bounded by

$$
\max \left\{v_{i+1}(u)-v_{i+1}\left(\frac{1}{6}\right), 1+v_{i+1}(x)-v_{i+1}\left(\frac{1}{6}\right)\right\} \leq 1,
$$

since both maximands are bounded by 1 ; the first because of the induction assumption, and the second because it is nonincreasing in $x$ and attains its maximum for $x=\frac{1}{6}$.

Finally, for $x \in\left[\frac{5}{6}, 1\right]$, the third integrand is bounded by

$$
\max \left\{v_{i+1}(u)-1-v_{i+1}(1-x), 0\right\} \leq 0,
$$

since $-v_{i+1}(1-x) \leq-v_{i+1}\left(\frac{1}{6}\right)$, and by the induction assumption we see that the left maximand $v_{i+1}(u)-1-v_{i+1}\left(\frac{1}{6}\right)$ is also nonpositive. So, at last we have

$$
v_{i}(u)-v_{i}\left(\frac{1}{6}\right) \leq \frac{5}{6}<1,
$$

and this completes the proof of condition (ii).

The last claim of Proposition 2 is that $\frac{1}{6} \leq f_{i, n}^{*}(y)$ for all $y \in[0,1]$ and all $1 \leq i \leq n-2$, $n \geq 3$. If $y \in\left[\frac{1}{6}, 1\right]$, this bound is trivial since $y \leq f_{i, n}^{*}(y)$ for all $1 \leq i \leq n$. If $y \in\left[0, \frac{1}{6}\right)$ then inequality (i) gives $1<v_{i+1, n}(y)-v_{i+1, n}\left(\frac{5}{6}\right)$ for all $1 \leq i \leq n-2$, so that the definition of $f_{i, n}^{*}(y)$ in (23) gives the required lower bound. This completes the proof of Proposition 2.

\subsection{Proof of the upper bound-the last step}

We now have all the tools that we need to prove that there is a constant $C<11-4 \sqrt{2} \sim 5.343$ such that

$$
\mathrm{E}\left[A_{n}^{o}\left(\pi_{n}^{*}\right)\right] \leq(2-\sqrt{2}) n+C \text { for all } n \geq 1 .
$$

We first note that the bound is trivial for $n=1$ and $n=2$. For $n \geq 3$, let $\left\{f_{1, n}^{*}, \ldots, f_{n, n}^{*}\right\}$ denote the optimal threshold functions determined by the recursive solution of the Bellman equation (22) for the finite-horizon problem with sample size $n$. We will use the first $n-2$ of these functions to construct a suboptimal selection policy for the geometric sample size problem. From the suboptimality of this policy, we will obtain an inequality that will lead to our upper bound. 


\subsection{Construction of a suboptimal policy for the infinite-horizon problem}

We now consider the infinite-horizon problem, and, as before, we let $\left\{X_{1}, X_{2}, \ldots\right\}$ denote the sequence of observations. Our selection process is as follows.

(a) We let $T_{0}$ denote the index of the first observation in the sequence that falls in the interval $\left[\frac{5}{6}, 1\right]$. We select that observation as the first element of our subsequence, and we set $Y_{T_{0}}=1-X_{T_{0}}$. We note that $Y_{T_{0}}$ is uniformly distributed on $\left[0, \frac{1}{6}\right]$.

(b) Next we use the functions $\left\{f_{1, n}^{*}, \ldots, f_{n-2, n}^{*}\right\}$ to decide which of the next $n-2$ observations are to be selected. Specifically, we make our $i$ th selection in the series if $X_{T_{0}+i} \geq$ $f_{i, n}^{*}\left(Y_{T_{0}+i-1}\right)$, where, as usual, the $Y_{T_{0}+i}$ are defined by the recursion

$$
Y_{T_{0}+i}= \begin{cases}Y_{T_{0}+i-1} & \text { if } X_{T_{0}+i}<f_{i, n}^{*}\left(Y_{T_{0}+i-1}\right) \\ 1-X_{T_{0}+i} & \text { if } X_{T_{0}+i} \geq f_{i, n}^{*}\left(Y_{T_{0}+i-1}\right)\end{cases}
$$

Here one should recall that by Proposition 2 we have $\frac{1}{6} \leq f_{i, n}^{*}\left(Y_{T_{0}+i-1}\right)$ for $1 \leq i \leq n-2$, so we have $0 \leq Y_{T_{0}+i} \leq \frac{5}{6}$ for $1 \leq i \leq n-2$.

(c) We will now show how our selection process can be repeated in a stationary way. For $k=0,1,2, \ldots$, we proceed as follows.

1. If $Y_{T_{k}+n-2} \in\left(\frac{1}{6}, \frac{5}{6}\right]$ then we let

$$
\tau_{k}=\inf \left\{i \geq 1: X_{T_{k}+n-2+i} \geq \frac{5}{6}\right\}
$$

and we select the observation $X_{T_{k}+n-2+\tau_{k}}$. We note that the random variable $Y_{T_{k}+n-2+\tau_{k}}=1-X_{T_{k}+n-2+\tau_{k}}$ is uniformly distributed on $\left[0, \frac{1}{6}\right]$.

2. If $Y_{T_{k}+n-2} \leq \frac{1}{6}$ then we simply let $\tau_{k}=0$, and we again note that $Y_{T_{k}+n-2+\tau_{k}}$ is uniformly distributed on $\left[0, \frac{1}{6}\right]$.

3. We set $T_{k+1}=T_{k}+n-2+\tau_{k}$ and $k=k+1$.

4. Just as in (b), we use the functions $\left\{f_{1, n}^{*}, \ldots, f_{n-2, n}^{*}\right\}$ to decide which observations to select from $\left\{X_{T_{k}+1}, X_{T_{k}+2}, \ldots, X_{T_{k}+n-2}\right\}$. At time $T_{k}+n-2$ we are left with some value $Y_{T_{k}+n-2}$, and we return to step 1 .

\subsection{Analysis of the policy}

The suboptimal policy we constructed provides us with an increasing sequence of stopping times $0<T_{0}<T_{1}<T_{2}<\cdots$ such that the times $\left\{T_{k}: k \geq 1\right\}$ are regeneration times for the process $\left\{Y_{i}: i \geq T_{0}\right\}$. Moreover, we also have an independent and identically distributed (i.i.d.) sequence of stopping times $\left\{\tau_{k}: k \geq 1\right\}$ with distribution

$$
\tau_{k} \stackrel{\mathrm{D}}{=} \begin{cases}0 & \text { if } Y_{T_{0}+n-2} \leq \frac{1}{6} \\ \inf \left\{i \geq 1: X_{i}>\frac{5}{6}\right\} & \text { if } Y_{T_{0}+n-2}>\frac{1}{6}\end{cases}
$$

The regeneration times $\left\{T_{k}: k \geq 1\right\}$ can be written as a function of the stopping times $\left\{\tau_{k}: k \geq\right.$ $1\}$; specifically, we have

$$
T_{k}=T_{0}+(n-2) k+\sum_{j=1}^{k} \tau_{j}
$$


For any pair $\left(T_{k}, Y_{T_{k}}\right), 1 \leq k<\infty$, the number $r\left(T_{k}, Y_{T_{k}}\right)$ of selections made from $\left\{X_{T_{k}+1}, \ldots, X_{T_{k}+n-2}\right\}$ is then given by the sum

$$
r\left(T_{k}, Y_{T_{k}}\right):=\sum_{i=1}^{n-2} \mathbf{1}\left(X_{T_{k}+i} \geq f_{i, n}^{*}\left(Y_{T_{k}+i-1}\right)\right) .
$$

For each $0<\rho<1$, the described selection process gives a feasible policy that lower bounds the expected length, $\mathrm{E}\left[A_{N}^{o}\left(\pi^{*}\right)\right]$, of the alternating subsequence selected by an optimal policy from a sample of geometric size.

Moreover, if, for discounting purposes, we view the number of selections $r\left(T_{k}, Y_{T_{k}}\right)$ as being counted all at time $T_{k}+n-2$, then we obtain a lower bound for the expected value achieved by our suboptimal policy. We therefore have the bound

$$
\mathrm{E}\left[\sum_{k=0}^{\infty} \rho^{T_{k}+n-2} r\left(T_{k}, Y_{T_{k}}\right)\right] \leq \mathrm{E}\left[A_{N}^{o}\left(\pi^{*}\right)\right]
$$

We now note that $T_{0}$ and $Y_{T_{0}}$ are independent, and we also note that, for each $k \geq 1$, the post- $T_{k}$ process $\left\{Y_{T_{k}+i}: i \geq 0\right\}$ is independent of $T_{k}$. Consequently, we have the factorization

$$
\mathrm{E}\left[\rho^{T_{k}+n-2} r\left(T_{k}, Y_{T_{k}}\right)\right]=\mathrm{E}\left[\rho^{T_{k}+n-2}\right] \mathrm{E}\left[r\left(T_{k}, Y_{T_{k}}\right)\right] \text { for all } k \geq 0,
$$

and, since $T_{k}$ is a regeneration epoch, we also have

$$
\mathrm{E}\left[r\left(T_{k}, Y_{T_{k}}\right)\right]=\mathrm{E}\left[r\left(T_{0}, Y_{T_{0}}\right)\right] \text { for all } k \geq 0 .
$$

For $Y_{T_{0}}=y \in\left[0, \frac{1}{6}\right]$, we recall identity (25) and observe that

$$
v_{1, n}(y)-2 \leq \mathrm{E}\left[r\left(T_{0}, Y_{T_{0}}\right) \mid Y_{T_{0}}=y\right],
$$

since the policy on the right-hand side agrees with the policy on the left-hand side for the first $n-2$ observations, and the policy on the right-hand side never selects the last two observations.

The monotonicity of $v_{1, n}(\cdot)$ and Proposition 2(ii) then give the lower bound

$$
\mathrm{E}\left[A_{n}^{o}\left(\pi_{n}^{*}\right)\right]-3=v_{1, n}(0)-3 \leq \mathrm{E}\left[r\left(T_{0}, Y_{T_{0}}\right) \mid Y_{T_{0}}=y\right] \text { for all } 0 \leq y \leq \frac{1}{6},
$$

so, by recalling that $0 \leq Y_{T_{0}} \leq \frac{1}{6}$ and taking total expectations, we see that

$$
\mathrm{E}\left[A_{n}^{o}\left(\pi_{n}^{*}\right)\right]-3 \leq \mathrm{E}\left[r\left(T_{0}, Y_{T_{0}}\right)\right] .
$$

Factorization (34) then gives the bound

$$
\mathrm{E}\left[\rho^{T_{k}+n-2}\right]\left(\mathrm{E}\left[A_{n}^{o}\left(\pi_{n}^{*}\right)\right]-3\right) \leq \mathrm{E}\left[\rho^{T_{k}+n-2} r\left(T_{k}, Y_{T_{k}}\right)\right] \quad \text { for all } k \geq 0 .
$$

If we now sum over $k$, and use representation (32) and the suboptimality condition (33), we have

$$
\left(\mathrm{E}\left[A_{n}^{o}\left(\pi_{n}^{*}\right)\right]-3\right) \mathrm{E}\left[\sum_{k=0}^{\infty} \rho^{T_{0}+(n-2)(k+1)+\sum_{j=1}^{k} \tau_{j}}\right] \leq \mathrm{E}\left[A_{N}^{o}\left(\pi^{*}\right)\right] .
$$

We now note that $T_{0}$ is also independent from the random variables $\left\{\tau_{k}: k \geq 1\right\}$, and we recall that the $\tau_{k}$ s are i.i.d., so

$$
\mathrm{E}\left[\sum_{k=0}^{\infty} \rho^{T_{0}+(n-2)(k+1)+\sum_{j=1}^{k} \tau_{j}}\right]=\mathrm{E}\left[\rho^{T_{0}}\right] \sum_{k=0}^{\infty} \rho^{(n-2)(k+1)} \mathrm{E}\left[\rho^{\tau_{1}}\right]^{k} .
$$


Since $x \mapsto \rho^{x}$ is convex, Jensen's inequality tells us that $\rho^{\mathrm{E} T_{0}} \leq \mathrm{E}\left[\rho^{T_{0}}\right]$ and that $\rho^{\mathrm{E} \tau_{1}} \leq \mathrm{E}\left[\rho^{\tau_{1}}\right]$, so we have

$$
\rho^{\mathrm{E} T_{0}+n-2} \sum_{k=0}^{\infty}\left(\rho^{n-2+\mathrm{E} \tau_{1}}\right)^{k} \leq \mathrm{E}\left[\rho^{T_{0}}\right] \sum_{k=0}^{\infty} \rho^{(n-2)(k+1)} \mathrm{E}\left[\rho^{\tau_{1}}\right]^{k} .
$$

The left-hand side is an easy geometric series, and by substitution in (35) we obtain the crucial bound

$$
\mathrm{E}\left[A_{n}^{o}\left(\pi_{n}^{*}\right)\right] \leq 3+\frac{1-\rho^{n-2+\mathrm{E} \tau_{1}}}{\rho^{\mathrm{E} T_{0}+n-2}} \mathrm{E}\left[A_{N}^{o}\left(\pi^{*}\right)\right] .
$$

From the explicit formula for $\mathrm{E}\left[A_{N}^{o}\left(\pi^{*}\right)\right]$ in Theorem 2 we then have

$$
\mathrm{E}\left[A_{n}^{o}\left(\pi_{n}^{*}\right)\right] \leq 3+\frac{\left(1-\rho^{n-2+\mathrm{E} \tau_{1}}\right)(3-2 \sqrt{2}-\rho+\rho \sqrt{2})}{\rho^{\mathrm{E} T_{0}+n-1}(1-\rho)} .
$$

The bound above holds for all $0<\rho<1$, so by letting $\rho \uparrow 1$ we obtain

$$
\mathrm{E}\left[A_{n}^{o}\left(\pi_{n}^{*}\right)\right] \leq 3+(2-\sqrt{2})\left(n-2+\mathrm{E} \tau_{1}\right)<(2-\sqrt{2}) n+11-4 \sqrt{2},
$$

since $\mathrm{E}\left[\tau_{1}\right]<6$. This completes the proof of the upper bound.

\section{Observations on methods and connections}

Our principal goal has been to provide a reasonably definitive solution of a concrete problem of sequential optimization. Still, the natural expectation is that the solution of such a problem should also offer some novel methodological perspective. Here we began by exploiting the well-known idea of passing to the infinite-horizon problem, but less commonly (and somewhat doggedly) we made the trek back from the infinite-horizon problem to the finite-horizon problem. In retrospect, that trek had elements of inevitability to it, but it also had surprises.

In a natural and easy way the policy for the infinite-horizon problem gave us a lower bound for the finite-horizon problem, but our first surprise was the discovery (at first numerically) that the lower bound was so close to optimal. There was also something natural about the upper bound for the finite-horizon problem, though at first we argued it by contradiction. The idea was that if we had a policy for the finite horizon that was 'too good' then we should be able to concatenate that policy to give a policy for the infinite-horizon problem that would do better than our known optimal policy. The resulting contradiction would then provide an upper bound.

This three-step process would seem to be applicable to many problems of optimal selection, though, from the details of our proof, it is clear that special features must be exploited. For example, without obtaining four relations in Lemma 2, we would not have been able to solve the infinite-horizon problem. Three of these relations were straightforward, but the critical fourth relation still seems 'lucky'. We are also fortunate that symmetry relations simplified our Bellman equations. These simplifications have an intuitive basis from the alternating nature of the problem, but it seems fortuitous that these relations could be made rigorous by inductions (of several kinds) on the Bellman equation.

There are many problems where we would like to go from the infinite-horizon problem to the finite-horizon problem, in particular that of the optimal online selection of a monotone subsequence from a sample of independent observations. Here one knows the asymptotic behavior of the means for both finite samples (see Samuels and Steele (1981)) and random samples_including geometric-sized samples (see Gnedin (1999), (2000)). Most notably, in the infinite-horizon case we have a precise understanding of the variance and even a central 
limit theorem (see Bruss and Delbaen (2001), (2004)). It would be quite interesting to know if such an analogous central limit theorem can be obtained under the finite-horizon formulation.

\section{References}

Bertsekas, D. P. And Shreve, S. E. (1978). Stochastic Optimal Control (Math. Sci. Eng. 139). Academic Press, New York.

Bruss, F. T. AND Delbaen, F. (2001). Optimal rules for the sequential selection of monotone subsequences of maximum expected length. Stoch. Process. Appl. 96, 313-342.

Bruss, F. T. and Delbaen, F. (2004). A central limit theorem for the optimal selection process for monotone subsequences of maximum expected length. Stoch. Process. Appl. 114, 287-311.

Gnedin, A. V. (1999). Sequential selection of an increasing subsequence from a sample of random size. J. Appl. Prob. 36, 1074-1085.

GNEDIN, A. V. (2000). Sequential selection of an increasing subsequence from a random sample with geometrically distributed sample-size. In Game Theory, Optimal Stopping, Probability and Statistics (IMS Lecture Notes Monogr. Ser. 35), Institute of Mathematical Statistics, Beachwood, OH, pp. 101-109.

Houdré, C. ANd Restrepo, R. (2010). A probabilistic approach to the asymptotics of the length of the longest alternating subsequence. Electron. J. Combinatorics 17, 19pp.

Samuels, S. M. and Steele, J. M. (1981). Optimal sequential selection of a monotone sequence from a random sample. Ann. Prob. 9, 937-947.

Stanley, R. P. (2007). Increasing and decreasing subsequences and their variants. In International Congress of Mathematicians, Vol. I, European Mathematical Society, Zürich, pp. 545-579.

STANLEY, R. P. (2008). Longest alternating subsequences of permutations. Michigan Math. J. 57, 675-687.

Stanley, R. P. (2010). A survey of alternating permutations. In Combinatorics and Graphs (Contemp. Math. 531), American Mathematical Society, Providence, RI, pp. 165-96.

Widom, H. (2006). On the limiting distribution for the length of the longest alternating sequence in a random permutation. Electron. J. Combinatorics $13,7 \mathrm{pp}$. 